\title{
Chapter 24: Addressing the Challenges of Institutional Adoption
}

\author{
Cassandra Colvin' ${ }^{1}$, Shane Dawson' ${ }^{1}$, Alexandra Wade², Dragan Gašević ${ }^{3}$ \\ 'Teaching Innovation Unit, University of South Australia, Australia \\ ${ }^{2}$ School of Health Sciences, University of South Australia, Australia \\ ${ }^{3}$ Schools of Education and Informatics, The University of Edinburgh, United Kingdom \\ DOI: $10.18608 / \mathrm{hla} 17.024$
}

\begin{abstract}
Despite increased funding opportunities, research, and institutional investment, there remains a paucity of realized large-scale implementations of learning analytics strategies and activities in higher education. The lack of institutional exemplars denies the sector broad and nuanced understanding of the affordances and constraints of learning analytics implementations over time. This chapter explores the various models informing the adoption of large-scale learning analytics projects. In so doing, it highlights the limitations of current work and proposes a more empirically driven approach to identify the complex and interwoven dimensions impacting learning analytics adoption at scale.
\end{abstract}

Keywords: Learning analytics adoption, learning analytics uptake, leadership

The importance of data and analytics for learning and teaching practice is strongly argued in the education policy and research literature (Daniel, 2015; Siemens, Dawson, \& Lynch, 2013). The insights into teaching, learning, student-experience, and management activities that learning analytics afford are touted to be unprecedented in scale, sophistication, and impact (Baker \& Inventado, 2014). Not only do learning analytics have the capacity to provide rich understanding of practices and activities occurring within institutions, they also have the potential to mediate and shape future activity through, for example, predictive modelling, personalization of learning, and recommendation systems (Conde \& Hernández-García, 2015).

Despite increased funding opportunities, research, and institutional investment, there remains a paucity of realized large-scale implementations of learning analytics strategies and activities in higher education (Ferguson et al., 2015), thus denying the sector broad and nuanced understanding of the affordances and constraints of learning analytics implementations over time. Part of the explanation for the lack of enterprise exemplars may lie in the relative nascency of learning analytics as a discipline and a perceived lack of time for learning analytics programs and implementations to fully develop and mature. However, this explanation does not adequately capture the complexity of issues mediating systemic uptake of learning analytics (Arnold, Lynch, et al., 2014; Ferguson et al., 2015; Macfadyen, Dawson, Pardo, \& Gašević, 2014). Although learning analytics is relatively new to higher education, we suggest there have been sufficient investments made in time and resources to realize the affordances such activities can bring to education at a whole-of-institution scale. Indeed, a small number of institutions have been able to implement large-scale learning analytics programs with demonstrable impact on their teaching and learning outcomes (Ferguson et al., 2015). However, these examples remain the exception in a sector where, for a large number of institutions, organizational adoption of learning analytics either remains a conceptual, unrealized aspiration or, where operationalized, is often narrow and limited in scope and impact (Ferguson et al., 2015).

A burgeoning body of conceptual literature has recently begun to explore this vexing issue (Arnold, Lonn, \& Pistilli, 2014; Arnold, Lynch, et al., 2014; Ferguson et al., 2015; Macfadyen et al., 2014; Norris, Baer, Leonard, Pugliese, \& Lefrere, 2008). This literature proffers multiple frameworks intended to capture, and elicit insight into, dimensions and processes mediating learning analytics adoption. In addition to aiding conceptual 
understanding, this literature also has a heuristic value, guiding institutions through implementation stages and considerations. Given the limited empirical research exploring learning analytics deployment (Ferguson et al., 2015), it is probable that many managers turn to this small body of conceptual literature for inspiration and insight when planning and administering learning analytics initiatives. The present chapter reviews this body of literature to glean from it not only insight into what it identifies as dimensions and processes important for effective institutional implementations of learning analytics, but also to gauge the merit of the models as guides for institutional managers. We then compare and contrast the findings of this review of the literature with those from a recent study that examined actual learning analytics implementations across a large cohort of Australian universities to proffer empirical understanding into the processes and factors affording them (Colvin et al., 2015).

\section{REVIEW OF CURRENT MODELS OF LEARNING ANALYTICS DEPLOYMENT}

Review of extant learning analytics implementation models and frameworks revealed three primary groups of literature: 1) those focused on the antecedents to learning analytics outcomes (learning analytics inputs models); 2) those focused on the outcomes of learning analytics (learning analytics outputs models); and 3) process models that sequentially map and operationalize tasks underpinning learning analytics implementations. An overview of these different models, and their conceptual and empirical contribution to understanding factors shaping institutional learning analytics implementations, follows.

\section{Learning Analytics Inputs Models}

Frameworks in this body of literature tend to present learning analytics implementations as a consequence of antecedent affordances incorporating dimensions such as leadership, governance, technology, capacity, and culture. Notable in this literature is the US-based EDUCAUSE Centre for Analysis and Research (ECAR; see ECAR, 2015) Analytics Maturity Index for Higher Education (Bichsel, 2012). Their model, informed by data elicited through surveys and focus group interviews with industry professionals, operationalizes learning analytics implementations across six dimensions of activity including culture, process, data/reporting/ tools, investment, expertise, and governance/infrastructure. Each input dimension is scaffolded across a continuum of five maturity levels designed to assist institutions in determining their level of progress within each level. The criticality of each input dimension for a successful learning analytics implementation is assumed.
Similar to the ECAR model is the Learning Analytics Readiness Instrument (LARI) (Arnold, Lonn, \& Pistilli, 2014; Oster, Lonn, Pistilli, \& Brown, 2016), a tool designed to assist institutions in assessing their level of "readiness" for analytics implementations. The original version of the instrument (Arnold, Lonn, \& Pistilli, 2014) identified five dimensions - 1) ability, 2) data, 3) culture and process, 4) governance and infrastructure, and overall 5) readiness perceptions - as essential for achieving "the optimal environment for learning analytics success" (p. 2), although it is unclear how the five elements were initially determined. A more recent factor analysis of survey data from 560 participants across 24 institutions was used to refine the LARI. The five dimensions were slightly altered, and their relative salience was revealed. However, salience was measured according to participant perception, and not against learning analytics implementations outcomes.

The Organizational Capacity Analytics Framework (Norris \& Baer, 2013) is also founded on insight gleaned from learning analytics specialists as to dimensions they consider important in shaping analytics adoption. The authors interviewed managers from 40 institutions in the United States, and data collected through these interviews led to the generation of five dimensions deemed to be critical organizational capacity factors. These dimensions were 1) technology infrastructure, 2) processes and practice, 3) culture and behaviours, 4) skills and values, and 5) leadership. Notable in their Organizational Capacity for Analytics Framework is the presentation of the dimensions as interconnected and overlapping, thereby highlighting their interdependent nature (Norris \& Baer, 2013, p. 31). While the framework operationalizes three maturity levels for each of the dimensions, it does not examine their relative salience.

Finally, Drachsler and Greller's model (referred to by the authors as an ontology; Drachsler \& Greller, 2012; Greller \& Drachsler, 2012) also captures the interdependent and recursive nature of dimensions mediating learning analytics implementations. General morphological analysis (cf. Ritchey, 2011, in Greller \& Drachsler, 2012) was applied to data solicited from media scanning, interviews with senior experts, and a cognitive mapping exercise. This model identifies six core activity areas as "critical" to "ensure an appropriate exploitation of learning analytics" (Drachsler \& Greller, 2012, p. 120): 1) competences, 2) constraints (privacy/ethics), 3) technologies, 4) education data, 5) objectives, and 6) stakeholders. While Drachsler and Greller (2012) deem each of these six dimensions to be "critical," they observe that their salience is not uniform, noting "some dimensions are vaguer than others" (p. 44). 
Common to many input models is the conceptualization of learning analytics implementations as non-linear, emergent from, and afforded by, the interplay of multiple, interconnected input dimensions. While often informed by opinion solicited through focus group and survey methods (Drachsler \& Greller, 2012; Greller \& Drachsler, 2012; Norris \& Baer, 2013; Oster et al., 2016), these models are essentially conceptual. They identify dimensions that institutional representatives perceive must be considered to mount an effective learning analytics implementation, yet they do not empirically interrogate the dimensions against actual learning analytics implementations. Similarly, while the input models suggest interrelationships between antecedent dimensions, the nature of these interrelationships, and their impacts on learning analytic implementation outputs, are also not empirically explored. Therefore, while the models offer leaders charged with the task of implementing learning analytics programs in their institutions insight into the antecedent dimensions necessary to effect an implementation, they present little guidance on how such implementations could look in action. Further, the relative salience of each dimension within the models is underexplored, limiting insight into how institutions might best prioritize actions and resources (although a limited number of models do accommodate gradations of maturity within each dimension; Arnold, Lonn, \& Pistilli, 2014; Bichsel, 2012; Norris \& Baer, 2013).

\section{Learning Analytics Outputs Models}

This second body of learning analytics models and frameworks defines and represents learning analytics implementations as a linear process, unfolding over time, and involving different levels of readiness and maturity. An early model in this literature that still appears to have resonance in the sector is Davenport and Harris's (2007) Analytics Framework, which conceptualizes analytics as a maturing process from query and reporting applications through to formal analytics functions such as forecasting and predictive modelling. Siemens, Dawson, and Lynch's (2013) Learning Analytics Sophistication Model integrates analytic capability and systems deployment along a continuum of increasing maturity. Five key stages of maturity are identified, each of these further operationalized into sample exemplars. For instance, an early stage deployment would feature basic reports and log data whereas a mature deployment would feature predictive models and personalized learning.

A primary benefit of outputs models is that they provide a means for institutions to objectively assess the maturity (or capacity) of their activities and processes against a matrix of desired outcomes. However, many outputs models are limited in scope and typically predicated on a uni- or bi-dimensional conceptual lens (such as the sophistication of analytic techniques employed). While outputs models advocate a vision of learning analytics implementations outcomes, they often fail to identify or critically examine all of the dimensions or mechanisms needed to generate the LA implementation outcomes they in fact advocate. Finally, a risk of many outputs models is that they conceptualize progression as a linear and hierarchical process, culminating in an "essentialized," perhaps even "utopian" vision of learning analytics, one that is typically conceptual, removed from context, possibly predicated on an assumed universality, and not necessarily capturing what might be possible or desirable within the scope of a particular institution's operating context.

\section{Process Models}

This third body of literature (Foreman, 2013a, 2013b; Norris \& Baer, 2013) sequentially maps key processes or "steps" underpinning learning analytics implementations. It focuses on the how of implementing a learning analytics program, rather than what the outcomes should look like (outputs model) or involve (inputs model). Process models are both linear (Foreman, 2013a, 2013b) or circuitous (Norris and Baer's Action Plan for Analytics, 2013) and are typically focused on specific elements within a broader learning analytics implementation (for instance, the implementation of a learning management system (LMS; Foreman, 2013a, 2013b), or strategy development (cf. Norris and Baer's Action Plan for Analytics, 2013). However, emerging literature (Ferguson et al., 2015) presents processual models that better reflect the breadth and complexity of learning analytics implementations, arguing that the insight this conceptualization affords is critical for institutions wishing to apply learning analytics "at scale." Most notable is Ferguson and colleagues' (2015) advocacy for adopting the RAPID Outcomes Mapping Approach (ROMA) for a learning analytics implementation. This model presents inputs dimensions in an operational sequence involving seven key steps from formulation of initial objectives through to final evaluation. However, Ferguson's model, in essence, is conceptually generated. While there is little evidence of the model's empirical validation, it has been applied as a lens to describe learning analytics implementations at universities in Australia and the UK, argued by the authors to demonstrate the model's potential guide and give "confidence" to institutions (Ferguson et al., 2015).

\section{WHAT DO THE MODELS TELL US?}

While reviewing these models affords insight into dimensions and processes that mediate learning analytics deployments, it also reveals the models' conceptual and operational limitations. These include their adop- 
tion of a limited, or unidimensional lens to scrutinize complex, multidimensional phenomena; their inability to integrate antecedent dimensions and outcomes in the one model; and their limited insight into the relative salience or criticality of each of the identified mediating dimensions. Simply put, while the models afford insight, they do not fully capture the breadth of factors that shape LA implementations, thus curtailing their ability to present managers with the nuanced, situated, fine-grained insight they require to guide them through learning analytics implementations.

Notwithstanding, a number of mediating dimensions, or elements, were found to be common to most models, suggesting them to be particularly salient. These included technological readiness, leadership, organizational culture, staff and institutional capacity, and strategy. Discussion surrounding how these dimensions are operationalized in the models follows.

\section{Technological Readiness}

As learning analytics is essentially grounded in the affordance of technology to offer access and insight into electronic data, it is not surprising that technology features in LA implementation literature as a "foundational element" (Arnold, Lynch, et al., 2014, p. 258; refer also to Greller \& Drachsler, 2012; Siemens \& Long, 2011). However, operationalizations of dimensions described as technological readiness vary across the models. For instance, some models emphasize the need for a robust technology infrastructure that can collect, store, and transform data (Arnold, Lynch, et al., 2014, p. 258), while others reinforce the need for integrated systems (Dawson, Heathcote, \& Poole, 2010; Siemens \& Long, 2011), appropriate analytics tools (Norris \& Baer, 2013), and security and privacy controls and processes (for instance, the ECAR Analytics Maturity Index for Higher Education Model in Bichsel, 2012). Empirically, the potentially militating role of technology as a constraining element in learning analytics implementations was noted in studies undertaken by Dawson and colleagues (Dawson et al., 2010; Macfadyen \& Dawson, 2012).

\section{Leadership}

The criticality of leadership for sustainable implementations of learning analytics at scale is well recognized conceptually (Arnold, Lonn, \& Pistilli, 2014; Arnold, Lynch, et al., 2014; Laferrière, Hamel, \& Searson, 2013; Norris \& Baer, 2013; Siemens et al., 2013) and empirically (Graham, Woodfield, \& Harrison, 2013; Norris \& Baer, 2013). This literature advocates the importance of "committed" and "informed" leadership ground in a "deep scholarly understanding" of learning analytics to facilitate uptake and integration (Arnold, Lynch, et al., 2014, p. 260). While there is an obvious need for committed senior leadership, particularly in projects of scale and complexity (Norris \& Baer, 2013), there is a lack of consensus and commentary regarding how such leadership is conceptualized. For example, Laferrière et al. (2013) operationalized leadership through a uni- or limited dimensionality lens. In contrast, Arnold, Lynch et al. (2014) recognize leadership as a multilayered, multidimensional phenomenon. Leadership is also operationalized in the literature as leadership style (Owston, 2013), or leadership behaviour and influence (Laferrière et al., 2013), while other literature refers to leadership's structure (Accard, 2015; Carbonell, Dailey-Hebert, \& Gijselaers, 2013) and strength (cf. Kotter \& Schlesinger, 2008, in Arnold, Lynch, et al., 2014). Gaining particular traction is research advocating complexity (Hazy \& Uhl-Bien, 2014) or distributed leadership models (Bolden, 2011) to aid analytics implementation and uptake.

\section{Organizational Culture}

Organizational culture, defined as an institution's "norms, beliefs and values" (Carbonell et al., 2013, p. 30), has also been identified as a key mediator of learning analytics implementations (Arnold, Lonn, \& Pistilli, 2014; Carbonell et al., 2013; Greller \& Drachsler, 2012; Macfadyen \& Dawson, 2012). Prominent in this literature is an emphasis on staff "awareness and acceptance of data" (Arnold, Lonn, \& Pistilli, 2014, p. 164), a recognition of the potentially militating influence of an institution's "historical pedagogical [and] socio-cultural assumptions" vis-à-vis educational practice (Arnold, Lynch, et al., 2014, p. 259), organizational "routines" (Carbonell et al., 2013, p. 29), and even staff anxiety regarding organizational, pedagogical, and IT education change (Houchin \& MacLean, 2005). Empirical insight into the impact of an insufficiently prepared and receptive organizational culture has been offered in Macfadyen and Dawson's (2012) research into a failed implementation at a large Canadian university. The researchers observed that the institution's failure to generate a shared, willing, and receptive appreciation of learning analytics potential was a key reason for the organization's failure to roll out a coherent and successful learning analytics strategy.

\section{Staff and Institutional Capacity}

"Optimal" (Greller \& Drachsler, 2012, p. 51) learning analytics outcomes are contingent on the ability of staff to effectively analyse, interpret, and meaningfully respond to analytics intelligence (Bichsel, 2012; Norris \& Baer, 2013). However, it cannot be assumed that stakeholders possess the necessary analytical or interpretive data skills demanded of learning analytics. Norris and Baer (2013) observe that "many institutional leaders overestimate their enterprise's capacity in data, information, and analytics" (p. 40). Drachsler and Greller's (2012) research into the dimensions required in learning analytics implementations distinguishes 
between hard and soft dimensions: soft dimensions refer to the "human factors" that shape learning analytics effectiveness, notably "competences and acceptance" (p. 43); "hard" dimensions refer to nonhuman, less subjective elements, including technology, data, and algorithms. Distinguishing between hard and soft dimensions highlights the need for institutions to consider learning analytics implementations as extending beyond technical, infrastructure issues, to include sociocultural concerns. The successful adoption of learning analytics requires capacity building across these two domains.

The Learning Analytics Readiness Instrument (LARI; Arnold, Lonn, \& Pistilli, 2014) introduces a different conceptualization of capacity: institutional readiness - that is, a measure of how "ready" an institution is to implement a learning analytics initiative. They operationalize readiness across five dimensions: 1) ability, 2) data, 3) culture and process, 4) governance and infrastructure, and 5) overall readiness perception. Their conceptualization highlights the multilayered nature of capacity, noting that it presents at macro (i.e., broad, whole of institution) and micro (the level of the individual stakeholder) levels. Finally, in contrast with the focus on technical, critical, and interpretative capacity, Siemens, Dawson, and Lynch (2013) remind us of the relationship between learning analytics and teaching and learning practice, suggesting that capacity should also encompass the ability of staff to effectively "link" pedagogy and analytics.

\section{Strategy}

Conceptual literature advocates the development of a clear vision and purpose of learning analytics through the development of policy and procedures. For example, Arnold, Lonn, and Pistilli's (2014) conceptually developed Learning Analytics Readiness Instrument (LARI) subsumes policy into a broader category of mediating dimensions labelled governance and infrastructure. Further, Norris and Baer's Organizational Capacity for Analytics model (2013) identifies "Processes and Practices" as one of five key mediating dimensions of organizational capacity for LA, operationalizing this as "routinized processes and workflows to leverage [...] analytics, actions, and interventions" (p. 31). However, and by contrast, empirical studies stress the importance of strategy setting, and emphasize the need for aligned policies and objectives (Macfadyen \& Dawson, 2012; Owston, 2013), Macfadyen and Dawson (2012) declaring, in their analysis of a failed learning analytics program, that the establishment of an organizational strategy and vision was "critical" (p. 150) for learning analytics implementations.

\section{In Sum}

This brief literature review found that learning analytics models can provide managers with valuable insight into processes and dimensions shaping learning analytics implementations. Specifically, five dimensions were frequently highlighted across multiple frameworks as having impact on learning analytics implementation outcomes: technological readiness, leadership, organizational culture, staff and institutional capacity for learning analytics, and learning analytics strategy. However, as we have noted, operationalizations of these dimensions varied across the literature. Furthermore, the models afforded little insight into the relative salience, or criticality, of the dimensions. We suggest that the differing conceptualizations and operationalizations of the dimensions referred to in the literature have the potential to mediate how institutions engage with and interpret the many learning analytics frameworks available to them.

Further, and significantly, the literature introduced in this review is predominantly conceptual. We argue that the lack of empirical research into learning analytics implementations has hindered our understanding of the processes and dimensions that mediate them. While conceptual literature affords insight, it risks presenting an idealized model of learning analytics that might not adequately capture its full complexity and nuance. Where empirical techniques have been employed (such as soliciting data through surveys and focus groups), there is little detail surrounding construct validity. Accordingly, relationships between the different dimensions in the models appear to be largely untested. As observed earlier in this chapter, the relative immaturity of learning analytics programs in higher education institutions contributes in part to this empirical paucity surrounding learning analytics. However, we argue that the burgeoning, albeit nascent implementations found across higher education institutions provide an opportunity to empirically scrutinize how learning analytics implementations are currently being performed and mediated in context.

\section{BRINGING IN THE EMPIRICAL}

Recent research based in Australia has sought to address this research gap. Colvin et al. (2015) undertook a large national study investigating learning analytics implementations across the Australian higher education sector. Data were solicited through qualitative interviews with senior leaders charged with responsibility for implementing learning analytics at 32 universities. Utilizing a mixed-method methodology, the study identified six dimensions (inductively generated) that had a statistically significant impact on learning analytics implementations (out of the 27 dimensions identified in the data). Largely reflective of prior literature, four of the dimensions found to have impact included effective 
and distributed stakeholder engagement, technological capacity, clear vision and strategy, and influential leadership. Two other dimensions were revealed, namely institutional context (including an institution's student and institutional profile) and conceptualization (how an institution constructed and understood learning analytics). The former of these dimensions, institutional context, reminds us that learning analytics is situated in practice, shaped by an array of social and institutional structural elements unique to each institution's context. By contrast, the "conceptualization" dimension related to an institution's underlying epistemological and ontological position vis-à-vis learning analytics implementations. While institutions were found to have diverging understandings, aspirations, and visions of learning analytics, relationships were found between how learning analytics was conceptualized by an institution and how it was actually implemented. Simply, the findings of this study suggest that how learning analytics is understood, and the meaning assigned to it, appears to shape how it is implemented. Further, and significantly, cluster analysis performed in the research suggested the emergence of two trajectories of learning analytics implementation in the Australian higher education context. Within each of the clusters, there was congruence in how the conceptualization, readiness (antecedent), and implementation dimensions were performed and experienced across institutions. One cluster appeared to privilege a more instrumental conceptualization and a retention, student at-risk operationalization of learning analytics. By contrast, institutions in the second cluster were also invested in retention-focused learning analytics activity, but supplemented this with activity aimed to elicit insight into, and inform teaching and learning. Colvin at al.'s (2015) finding that there appear to be two diverging patterns of learning analytics implementations emerging in the Australian higher education context challenges the largely essentialist and positivist ontological and epistemological assumptions that underpin many extant learning analytics implementation frameworks (cf. Davenport \& Harris, 2007).

\section{Learning Analytics Implementations as Iterative, Dynamic, and Sustainable}

Based on these findings, Colvin et al. (2015) generated a model of strategic capability that presents learning analytics as a situated, multidimensional, dynamic, and emergent response to inter-relationships between six mediating dimensions; these not only afford and enable learning analytics implementations, but also recursively shape each other over time (Figure 24.1).

Figure 24.1 presents Colvin et al.'s Model of Strategic Capability learning analytics implementations that represents the phenomena as complex, dynamically interconnected, and temporal, and suggests that actual performance of learning analytics implementations will in turn generate future capacity. In this respect, and observed by the authors, the tenets of the Minimal Viable Product (MVP) (Münch et al., 2013), and the Rapid Innovation Cycle (Kaski, Alamäki, \& Moisio, 2014), with their advocacy for an ongoing, iterative, recursive, processual approach to product development and implementation, have traction in the learning analytics implementation space and are recommended to institutional leaders as possible implementation paradigms.

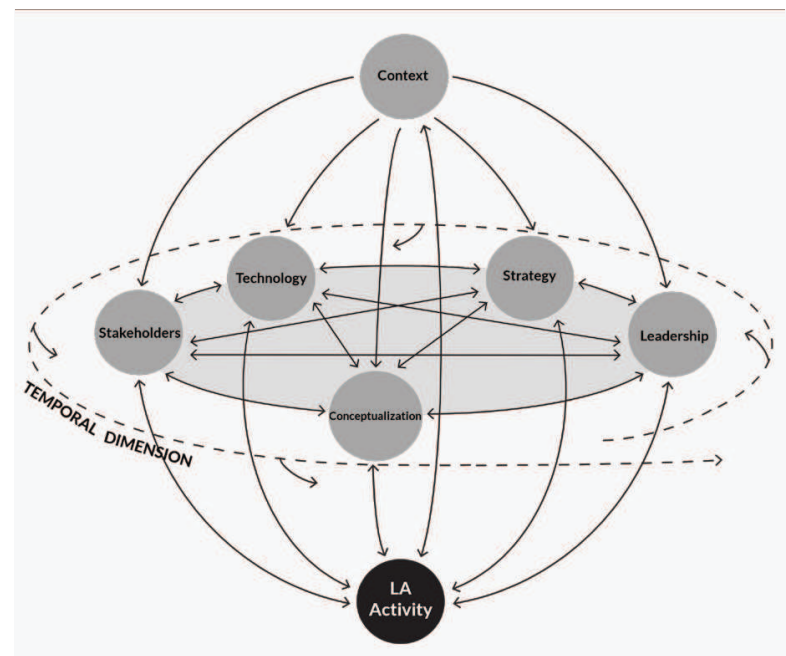

Figure 24.1. Model of Strategic Capability (Colvin et al., 2015, p. 28).

\section{CONCLUSION}

Colvin et al.'s (2015) work makes important empirical and methodological contributions to the research literature on learning analytics implementations. First, it provides empirical insights into the relationships between antecedents (affordances) of learning analytics implementations and their outcomes (that is, how they looked). Soliciting participants' meanings and understandings of actual learning analytics and learning analytics implementations provided finegrained, nuanced insight into the varied ways that institutional leaders conceptualized learning analytics implementations, and allowed relationships between these conceptualizations and actual operationalizations to be revealed.

The conceptualization and analysis of learning analytics implementations in Colvin et al.'s (2015) research as multidimensional phenomena resonates with tenets of emerging learning analytics implementation literature (cf. Ferguson et al., 2015; Greller \& Drachsler, 2012), and the Model of Strategic Capability generated by their research offers a rich, holistic, systemic conceptual- 
ization of learning analytics as a temporal, situated, dynamic consequence of multiple, intersecting, interdependent factors. Of particular significance though is the empirical insight Colvin et al. (2015) afford into the relative salience of the primary sociocultural, technical, and structural factors mediating learning analytics implementations.

Colvin et al.'s (2015) presentation of learning analytics diverges from many extant models, which often frame learning analytics as linear and/or unidimensional phenomena. We suggest that these latter conceptualizations, through their reductionist orientation, do not have the potential to fully capture the complexity, breadth, or disruption of learning analytics implementations, and may be inadvertently militating against the adoption and development of sustainable and effective learning analytics practices and strategies. By contrast, Colvin et al.'s (2015) findings remind us that learning analytics implementations are complex, shaped by interdependent "soft and hard" dimensions (Greller \& Drachsler, 2012), and have the potential to challenge and disrupt traditional management and organizational structures in universities. Their research provides institutional learning analytics managers with an empirically derived conceptual framework that highlights the complexity of learning analytics implementations, recognizes the mediating role of context, and can facilitate intra- and inter-institutional evaluation of learning analytics strategies and priorities.

It must be noted that Colvin et al.'s (2015) research has limitations: its data were primarily qualitative, and gleaned from a relatively small sample of institution participants $(n=32)$ located in one higher education context (Australia). Therefore, any direct application of the findings to alternate higher education contexts should be undertaken with some caution. Further, most institutions were found to be in the very early stages of their learning analytics implementations: their programs were embryonic and still developing. Relationships reported between antecedent dimensions and outcomes are therefore to be interpreted within these temporal constraints. It is recommended that further empirical analyses of learning analytics implementations are conducted over time that allow more nuanced insight into this critical area. Notwithstanding, the findings of Colvin et al. (2015) offer an analytics framework that grounds learning analytics implementations in the tenets of multidimensionality and complexity that should have resonance with the broader analytics community.

\section{REFERENCES}

Accard, P. (2015). Complex hierarchy: The strategic advantages of a trade-off between hierarchical supervision and self-organizing. European Management Journal, 33(2), 89-103.

Arnold, K. E., Lonn, S., \& Pistilli, M. D. (2014). An exercise in institutional reflection: The learning analytics readiness instrument (LARI). Proceedings of the $4^{\text {th }}$ International Conference on Learning Analytics and Knowledge (LAK '14), 24-28 March 2014, Indianapolis, IN, USA (pp. 163-167). New York: ACM.

Arnold, K. E., Lynch, G., Huston, D., Wong, L., Jorn, L., \& Olsen, C. W. (2014). Building institutional capacities and competencies for systemic learning analytics initiatives. Proceedings of the $4^{\text {th }}$ International Conference on Learning Analytics and Knowledge (LAK '14), 24-28 March 2014, Indianapolis, IN, USA (pp. 257-260). New York: ACM.

Baker, R., \& Inventado, P. (2014). Educational data mining and learning analytics. In J. A. Larusson \& B. White (Eds.), Learning analytics (pp. 61-75). New York: Springer.

Bichsel, J. (2012). Analytics in higher education: Benefits, barriers, progress and recommendations. Louisville, CO: EDUCAUSE Center for Applied Research.

Bolden, R. (2011). Distributed leadership in organizations: A review of theory and research. International Journal of Management Reviews, 13(3), 251-269.

Carbonell, K. B., Dailey-Hebert, A., \& Gijselaers, W. (2013). Unleashing the creative potential of faculty to create blended learning. The Internet and Higher Education, 18, 29-37.

Colvin, C., Rogers, T., Wade, A., Dawson, S., Gašević, D., Buckingham Shum, S., . . Fisher, J. (2015). Student retention and learning analytics: A snapshot of Australian practices and a framework for advancement. Canberra, ACT: Australian Government Office for Learning and Teaching. 
Conde, M. Á., \& Hernández-García, Á. (2015). Learning analytics for educational decision making. Computers in Human Behavior, 47, 1-3.

Daniel, B. (2015). Big data and analytics in higher education: Opportunities and challenges. British Journal of Educational Technology, 46(5), 904-920.

Davenport, T. H., \& Harris, J. G. (2007). Competing on analytics: The new science of winning. Boston, MA: Harvard Business School Press.

Dawson, S., Heathcote, L., \& Poole, G. (2010). Harnessing ICT potential. International Journal of Educational Management, 24(2), 116-128.

Drachsler, H., \& Greller, W. (2012). The pulse of learning analytics understandings and expectations from the stakeholders. Proceedings of the $2^{\text {nd }}$ International Conference on Learning Analytics and Knowledge (LAK '12), 29 April-2 May 2012, Vancouver, BC, Canada (pp. 120-129). New York: ACM.

ECAR-ANALYTICS Working Group. (2015). The predictive learning analytics revolution: Leveraging learning data for student success ECAR working group paper. Louisville, CO: EDUCAUSE.

Ferguson, R., Macfadyen, L., Clow, D., Tynan, B., Alexander, S., \& Dawson, S. (2015). Setting learning analytics in context: Overcoming the barriers to large-scale adoption. Journal of Learning Analytics, 1(3), 120-144.

Foreman, S. (2013a). Five steps to evaluate and select an LMS: Proven practices. Learning Solutions Magazine. http://www.learningsolutionsmag.com/articles/1181/five-steps-to-evaluate-and-select-an-lms-provenpractices.

Foreman, S. (2013b). The six proven steps for successful LMS implementation. Learning Solutions Magazine. http://www.learningsolutionsmag.com/articles/1214/the-six-proven-steps-for-successful-lms-implementation-part-1-of-2.

Graham, C. R., Woodfield, W., \& Harrison, J. B. (2013). A framework for institutional adoption and implementation of blended learning in higher education. The Internet and Higher Education, 18, 4-14.

Greller, W., \& Drachsler, H. (2012). Translating learning into numbers: A generic framework for learning analytics. Journal of Educational Technology \& Society, 15(3), 42-57.

Hazy, J. K., \& Uhl-Bien, M. (2014). Changing the rules: The implications of complexity science for leadership research and practice. In D. V. Day (Ed.), Oxford Handbook of Leadership and Organizations. Oxford, UK: Oxford University Press.

Houchin, K., \& MacLean, D. (2005). Complexity theory and strategic change: An empirically informed critique. British Journal of Management, 16(2), 149-166.

Kaski, T., Alamäki, A., \& Moisio, A. (2014). A multi-discipline rapid innovation method. Interdisciplinary Studies Journal, 3(4), 163.

Kotter, J. P., \& Schlesinger, L. A. (2008). Leading Change. Boston, MA: Harvard Business Review Press.

Laferrière, T., Hamel, C., \& Searson, M. (2013). Barriers to successful implementation of technology integration in educational settings: A case study. Journal of Computer Assisted Learning, 29(5), 463-473.

Macfadyen, L., \& Dawson, S. (2012). Numbers are not enough. Why e-learning analytics failed to inform an institutional strategic plan. Educational Technology \& Society, 15(3), 149-163.

Macfadyen, L., Dawson, S., Pardo, A., \& Gašević, D. (2014). Embracing big data in complex educational systems: The learning analytics imperative and the policy challenge. Research E Practice in Assessment, 9(2), 17-28.

Münch, J., Fagerholm, F., Johnson, P., Pirttilahti, J., Torkkel, J., \& Jäarvinen, J. (2013). Creating minimum viable products in industry-academia collaborations. In B. Fitzgerald, K. Conboy, K. Power, R. Valerdi, L. Morgan, \& K.-J. Stol (Eds.), Lean Enterprise Software and Systems (Vol. 167, pp. 137-151). Springer Berlin Heidelberg.

Norris, D., Baer, L., Leonard, J., Pugliese, L., \& Lefrere, P. (2008). Action analytics: Measuring and improving performance that matters in higher education. EDUCAUSE Review, 43(1), 42. 
Norris, D. M., \& Baer, L. L. (2013). Building organizational capacity for analytics. Louisville, CO: EDUCAUSE.

Oster, M., Lonn, S., Pistilli, M. D., \& Brown, M. G. (2016). The learning analytics readiness instrument. Proceedings of the $6^{\text {th }}$ International Conference on Learning Analytics and Knowledge (LAK '16), 25-29 April 2016, Edinburgh, UK (pp. 173-182). New York: ACM.

Owston, R. (2013). Blended learning policy and implementation: Introduction to the special issue. The Internet and Higher Education, 18, 1-3.

Ritchey, T. (2011). General morphological analysis: A general method for non-quantified modelling. In T. Ritchey (Ed.), Wicked Problems: Social Messes. http://www.swemorph.com/pdf/gma.pdf

Siemens, G., Dawson, S., \& Lynch, G. (2013). Improving the quality and productivity of the higher education sector: Policy and strategy for systems-level deployment of learning analytics. Sydney, Australia: Australian Government Office for Teaching and Learning.

Siemens, G., \& Long, P. (2011). Penetrating the fog: Analytics in learning and education. EDUCAUSE Review, 46(5), 30. 